\title{
Modelo de gestión del conocimiento para pymes, basado en el sistema de gestión de la calidad y la gestión documental
}

\author{
Knowledge management model for SMEs, based on the quality \\ management system and document management
}

\section{Modelo de gestão do conhecimento para as PME, fundamentado no sistema de gestão da qualidade e na gestão de documentos}

Guillermo Peña Guarín *

Universidad Santo Tomás

Martha Lucia Castro Rojas"**

Icontec

María Janeth Álvares Álvarez" "***

Universidad de la Salle

Cómo citar este artículo: Peña Guarin, G., Castro Rojas, M.L y Álvarez Álvarez, M. (2020). Modelo de gestión del conocimiento para pymes, basado en el sistema de gestión de la calidad y la gestión documental. Signos, Investigación en Sistemas de Gestión, 12(2), 127-147. Dor: https://doi.org/10.15332/24631140.5941

\footnotetext{
Artículo de reflexión del grupo conformado para la investigación que se desarrolla entre la Universidad de La Salle, la Universidad Santo Tomás y el Instituto Colombiano de Normas Técnicas (Icontec).

** Guillermo Peña Guarín: Ingeniero químico; magíster en Calidad y Gestión Integral; Profesor Investigador de la Universidad Santo Tomás. Auditor de sistemas de gestión. Consultor organizacional. Bogotá (Colombia).Correo electrónico: guillermo.pena@usantotomas.edu.co

*** Martha Lucía Castro Rojas: Ingeniera química; especialista en Ingeniería Ambiental; jefe de Innovación y Cooperación de Icontec, Bogotá (Colombia). Correo electrónico: mcastro@icontec.org

**** María Janneth Álvarez: Bibliotecóloga y archivista; magíster en Docencia; profesora e investigadora de la Universidad de La Salle, Bogotá (Colombia). Correo electrónico:mjalvarez@unisalle.edu.co.
} 


\section{RESUMEN}

Algunos estudios indican que el conocimiento que necesitan las organizaciones para mejorar su desempeño se puede administrar de una manera más eficaz cuando se articula con la gestión de la calidad y con la gestión documental. Aplicando un método cualitativo, basado en una revisión de literatura y la identificación de prácticas de gestión documental en pymes seleccionadas, se elabora un modelo para la gestión del conocimiento que integra la gestión del conocimiento con el sistema de gestión de la calidad y con la gestión documental, según las normas técnicas NTC ISO 9001:2015 y NTC ISO 33001:2011, respectivamente. Este modelo articula el conocimiento capturado del exterior con el creado en la organización, con el fin de facilitar su gestión bajo las condiciones limitadas de las pymes.

Palabras clave: Gestión del conocimiento, gestión de la calidad, gestión

\section{ABSTRACT}

Some studies show that the knowledge that organizations need to improve their performance can be managed more effectively when articulated with quality management and document management. A model for knowledge management was developed applying a qualitative method, based on a literature review and the identification of document management practices in selected SMEs, that integrates knowledge management with the quality management system and with document management, according to the technical standards NTC ISO 9001:2015 and NTC ISO 33001:2011 respectively. This model integrates knowledge captured from outside with that created in the organization, in order to facilitate its management under the limited conditions of SMEs.
Keywords: Knowledge management, quality management, document management, ISO 9001, SME.

\section{RESUMO}

Alguns estudos apontam que o conhecimento de que as organizações necessitam para melhorar o seu desempenho pode ser gerido de forma mais eficaz quando articulado com a gestão da qualidade e a gestão documental. Aplicando um método qualitativo, baseado na revisão bibliográfica e na identificação de práticas de gestão documental nas PME selecionadas, foi desenvolvido um modelo de gestão do conhecimento que integra a gestão do conhecimento com o sistema de gestão da qualidade e com a gestão documental, de acordo com as normas técnicas NTC ISO 9001:2015 e NTC ISO 33001:2011, respetivamente. Este modelo combina os conhecimentos adquiridos do exterior com os adquiridos na organização, a fim de facilitar a sua gestão nas condições limitadas das PME.

Palavras-chave: Gestão do conhecimento, gestão da qualidade, gestão documental, ISO 9001, PME.

\section{INTRODUCCIÓN}

Tal como lo señala Flaherty (2001), la tecnología es el motor del cambio y el conocimiento (es decir, la información en acción) es su combustible; por lo tanto, el ritmo de todo cambio se puede observar en el avance de las herramientas tecnológicas y en la calidad del conocimiento que está presente en cada momento de la historia. Esta premisa la complementa Xavier Ferrás (2016), líder reconocido internacionalmente en temas de innovación, cuando explica las cuatro revoluciones industriales así: 
La primera es la energética (desde la máquina de vapor hasta la llegada de la electricidad). La segunda es la de la gestión (con la producción en masa y la eclosión de las ciencias de la administración). La tercera es la de la informática (la era del silicio, desde los ordenadores de válvulas de vacío hasta los smartphones). Pero la cuarta revolución industrial no será sólo la 'industria 4.0' será la revolución del conocimiento, en todas sus dimensiones. La información ya no es poder. El conocimiento lo es. (p. 1)

La cuarta revolución industrial estará marcada por la reunión de lo físico con lo digital y lo biológico, a través del uso intensivo de Internet, de la digitalización y de la comunicación en línea. Esta situación es particularmente retadora para las pymes, que en cualquier economía representan el mayor número de unidades económicas y generan aportes importantes al empleo, y que tendrán que acudir a la gestión del conocimiento si quieren sobrevivir.

Por lo tanto, resulta apropiado plantearse cómo pueden las pymes gestionar el conocimiento necesario para competir en un entorno globalizado, tecnológicamente acelerado y con las limitaciones de recursos que las caracterizan. De acuerdo con Álvarez-Álvarez, Castro-Rojas y Peña (2013):

La importancia estratégica que tienen los datos, la información y el conocimiento se puede aprovechar mejor, si de manera consciente la organización articula el sistema de gestión de la calidad, orientado a mejorar la relación con los clientes, con la gestión documental, orientada a capturar, organizar y facilitar los documentos que contienen los datos y la información, con la gestión del conocimiento, que se enfoca en encontrar y poner a disposición de la organización, el conocimiento que requiere para mejorar su desempeño. (p. 117)

Esta premisa se constituye en un insumo relevante para la elaboración del modelo propuesto, por lo que se decide tomar como plataforma o fundamento para ello el sistema de gestión de la calidad, tal como se plantea en la norma técnica internacional NTC ISO 9001 Sistemas de gestión de la calidad. Requisitos (Instituto Colombiano de Normas Técnicas y Certificación [Icontec], 2015), articulado con el sistema de gestión documental, establecido en la norma técnica internacional Iso 30301 Information and documentation. Management system for records (International Organization for Standardization [Iso], 2011).

El modelo Iso 9001 es conocido y ha sido ampliamente implementado por las organizaciones latinoamericanas y colombianas (ISO, 2015); este modelo promueve que el sistema de gestión de la calidad tenga un enfoque basado en procesos, es decir, que la gestión sea horizontal, cruzando las barreras entre diferentes unidades funcionales y unificando sus esfuerzos hacia las metas principales de la organización (ISO, 2003). También insiste en que durante la planificación y la ejecución se promueva una actitud preventiva, mediante la identificación y análisis de riesgos y oportunidades.

La orientación hacia los procesos que postula el modelo Iso 9001 implica la determinación de los procesos, de su secuencia y de sus interacciones, con el fin de alcanzar los objetivos previstos en el direccionamiento estratégico de la organización; la gestión de estos procesos y del sistema de gestión que conforman se puede hacer mediante el uso del ciclo de la mejora o ciclo PHVA (por planificar, hacer, verificary actuar), que se puede describir así: hay que planificar lo qué se quiere lograr y entender para qué se quiere lograr, además de organizar el tiempo, estimar los recursos que se necesitan y consignarlo todo en un plan; capacitar a las personas en el plan y enseñarles a hacer bien lo planificado; posteriormente, se debe verificar periódicamente si se está cumpliendo lo planificado y, de acuerdo con el resultado de la verificación, hay que actuar en consecuencia.

Por otra parte, la norma técnica Iso 30301 (ISO, 2011) hace referencia a la integralidad que debe existir en los procesos y controles para los documentos utilizados en las organizaciones desde el momento de su creación, durante el tiempo que deben permanecer accesibles 
para facilitar su consulta durante su uso en la toma de decisiones y en la conservación de estos en el mediano o largo plazo, de manera que se garanticen su legibilidad y autenticidad.

En el marco de las anteriores consideraciones, este estudio propone la elaboración de un modelo para facilitarle a las pymes la identificación y la gestión del conocimiento necesario para llevar a cabo sus operaciones en condiciones controladas, apoyándose en la gestión de la calidad y la gestión documental. El modelo debe orientar la gestión del conocimiento en cuanto a su reconocimiento, transmisión, utilización y conservación, así como en relación con su transformación en una plataforma que favorezca la sostenibilidad en el tiempo de la organización y que sea la base para producir innovación.

El modelo propuesto se apoya en modelos de gestión del conocimiento previos, como los que se analizan más adelante, pero sin las complejidades que algunos de estos poseen, para hacerlo accesible a las pymes.

\section{METODOLOGÍA}

Se aplicó un método cualitativo basado, por una parte, en una revisión de literatura sobre las teorías, modelos e iniciativas más aplicadas para la gestión del conocimiento en organizaciones, a partir de estudios realizados en los últimos quince años; se identificaron cinco teorías principales y los modelos más aplicados que se derivan de dichas teorías, analizando su aplicabilidad a las pymes. Por otra parte, se identificaron prácticas reales de gestión del conocimiento y de la documentación en pymes seleccionadas, a través de una entrevista semiestructurada que cubría las categorías de archivo, gestión documental, gestión de la calidad y gestión del conocimiento. Las entrevistas se aplicaron como parte del trabajo de grado de estudiantes del programa Sistemas de Información y Documentación de la Universidad de La Salle, y se llevaron a cabo en empresas pymes de de la siguiente manera:

- Diana Milena Rodríguez Benavides entrevistó una muestra de 19 pequeñas empresas de los sectores de servicios, industrial y comercio del municipio de Soacha.

- Adriana Castillo Aunta aplicó 5 entrevistas a pequeñas empresas de los sectores de servicios, industrial y comercio del municipio de Mosquera.

- Andrea Yesenia Martínez Cely aplicó la entrevista en 8 laboratorios clínicos del barrio Olaya de Bogotá.

- José Alexander Barrera Ruíz entrevistó 15 panaderías en Bogotá.

La selección de las muestras tuvo como punto de partida el registro mercantil de los respectivos municipios para ubicar a las pymes, y se utilizó el criterio de muestreo por conveniencia dada la dificultad para aceptar la entrevista por parte de las organizaciones.

Los resultados se analizaron mediante la elaboración de redes de sentido y mapas conceptuales, con el fin de organizar e interpretar las respuestas y encontrar conexiones entre las categorías propuestas.

\section{RESULTADOS}

En su libro Post-capitalist Society, Drucker (1994) establece que el recurso básico de la nueva economía no es ni la tierra, ni el trabajo, ni el capital, sino el conocimiento. La tierra, el trabajo y el capital no desaparecen, pero se vuelven secundarios. El valor se genera ahora por la productividad y la innovación, resultado de aplicar el conocimiento al trabajo. Asimismo, Drucker (1992) establece tres prácticas que deben abordar las organizaciones en esta nueva realidad: la primera es un proceso de mejoramiento continuo - lo que los japoneses llaman kaizen-; la segunda tiene que ver con aprender a 
explotar el conocimiento como fuente de generación de riqueza; finalmente, la tercera indica que cada organización debe aprender a innovar con procesos ordenados y sistemáticos basados en el conocimiento.

Para sobrevivir en el entorno actual, las organizaciones deben gestionar en forma coherente el conocimiento y, de esa manera, mitigar los riesgos a los que se encuentran expuestas en las relaciones con sus clientes y otras partes interesadas; los sistemas de gestión basados en normas técnicas internacionales pueden utilizarse para articular dicho conocimiento y orientarlo eficazmente a la mitigación de sus riesgos (Peña, 2017).

Las pymes constituyen en cualquier país el grupo predominante de empresas y, generalmente, superan el 95\% de las unidades económicas, contribuyendo significativamente a la creación de empleo y de riqueza, y satisfaciendo necesidades en mercados que son poco atractivos para las empresas más grandes.

En Colombia las empresas se clasifican —de acuerdo con la Ley 590 de 2000 y la Ley 905 de 2004- en micro, pequeñas, medianas y grandes empresas; el término pymes hace referencia al grupo de empresas pequeñas y medianas con activos totales superiores a 500 y hasta 30.000 salarios mínimos mensuales legales vigentes (SMMLV). De acuerdo con la Organización para la Cooperación y el Desarrollo Económicos (OECD, 2014), las pymes comprenden entre el $60 \%$ y $70 \%$ de la fuerza laboral de los países pertenecientes al acuerdo. El Banco Mundial (2015) señala que las pymes contribuyen con más del $60 \%$ de los empleos formales y con más del $40 \%$ del producto interno bruto de las economías emergentes. Según la revista Dinero ("Estas son las pymes...", 2017), en Colombia las pymes generan el $80 \%$ del empleo y las 1.000 más grandes — clasificadas por sus ventas - facturaron en el $2016 \$ 19$ billones de pesos, que equivalen a más de 6.500 millones de dólares; de las 25.000 empresas que reportan sus estados financieros a la Superintendencia de Sociedades, unas 20.000 son pymes. Sin embargo, y de acuerdo con la misma publicación, una pyme colombiana genera cinco veces menos valor agregado que una gran empresa.

La Asociación Nacional de Instituciones Financieras (ANIF) lleva a cabo semestralmente desde el 2006 la Gran Encuesta Pyme (GEP), a una muestra de más de 1.800 pymes de los sectores de industria, comercio y servicios; los resultados de la encuesta se resumen en el Indicador Pyme Anif (IPA) que permite visualizar el clima económico para las pymes, y que compara las variaciones semestrales en cuatro campos: 1) la situación económica actual, 2) el volumen de ventas, 3) las expectativas de desempeño y 4) las expectativas de ventas. La medición para el segundo semestre de 2017 arrojó un IPA de 54, valor por debajo del rango de entre 55 y 75 que se considera como un "clima económico bueno", lo que es consistente con la desaceleración económica del país, y que significa mayores retos para la supervivencia de las pymes al tener que enfrentar un ambiente turbulento (ANIF, 2017a).

Algunas de las características que diferencian a las pymes de grandes organizaciones son, entre otras, las siguientes (Rodríguez, 2015):

- Administración empírica. Los administradores de las pymes suelen ser sus propietarios, emprendedores que desconocen los fundamentos del desarrollo organizacional y, por lo tanto, copian modelos de negocio que han sido probados en otros campos o proceden intuitivamente para la toma de decisiones.

- Organización informal. La estructura de funciones y relaciones entre las personas es voluble, surge espontáneamente y no se documenta; al carecer de planificación, se ajusta según la dinámica de la empresa, creando duplicidades, inconsistencias y vacíos en las funciones.

Inestabilidad económica. La inestabilidad económica se determina por variaciones grandes en el flujo de ingresos que se originan por fluctuación en 
la comercialización de los productos y servicios, los cuales inciden negativamente sobre el flujo de caja, los pagos a proveedores y empleados, y los excedentes.

- Falta de conocimiento. La falta de conocimiento puede afectar tanto las actividades misionales como los aspectos administrativos y financieros de la organización; no se identifica el conocimiento necesario para operar eficaz y eficientemente, ni cómo se debe proteger, transmitir 0 incrementar dicho conocimiento.

En un estudio anterior (Álvarez-Álvarez et al., 2013), se estableció la convergencia entre los enfoques de la gestión del conocimiento, de la calidad y documental con el propósito de configurar un modelo de gestión del conocimiento. Este estudio mostró que el eje articulador entre la gestión del conocimiento, la gestión de la calidad y la gestión documental deberían ser los procesos que define el sistema de gestión de la calidad. También se comprobó que los datos, la información y el conocimiento se puede aprovechar mejor si la organización articula de manera consciente el sistema de gestión de la calidad —orientado a mejorar la relación con los clientes—, la gestión documental — dirigida a capturar, organizar y facilitar el flujo de los documentos que contienen los datos y la información-y la gestión del conocimiento —enfocada en encontrar y poner a disposición de la organización el conocimiento que requiere para mejorar su desempeño-(Álvarez-Álvarez et al., 2013). La gestión de la calidad y la gestión documental contienen elementos que fomentan la cultura por medio de la toma de conciencia y la comunicación interna. También convergen en el proceso de captura de conocimiento. Por su parte, la gestión documental permite soportar el conocimiento, preservándolo y facilitando su transmisión a quienes deben utilizarlo.

La norma técnica internacional NTC ISO 9001:2015, ampliamente utilizada por organizaciones en todo el mundo, propone unos requerimientos mínimos para implementar un sistema de gestión de la calidad (Icontec, 2015) y promueve que las organizaciones adopten un modelo de gestión por procesos, que se orienta a incrementar la satisfacción de los clientes y de otras partes interesadas, mediante el cumplimiento de sus requisitos. La comprensión y gestión de los procesos que se interrelacionan constituyendo un sistema contribuye al logro de los resultados previstos y, al controlar y regular las interrelaciones e interdependencias entre los procesos del sistema, se puede mejorar el desempeño global de la organización. La estructura general de esta norma técnica adopta el ciclo PHVA y se representa según la figura 1, en la que los números corresponden a capítulos de la norma. 
Figura 1. Estructura genérica de la norma técnica ISO 9001:2015

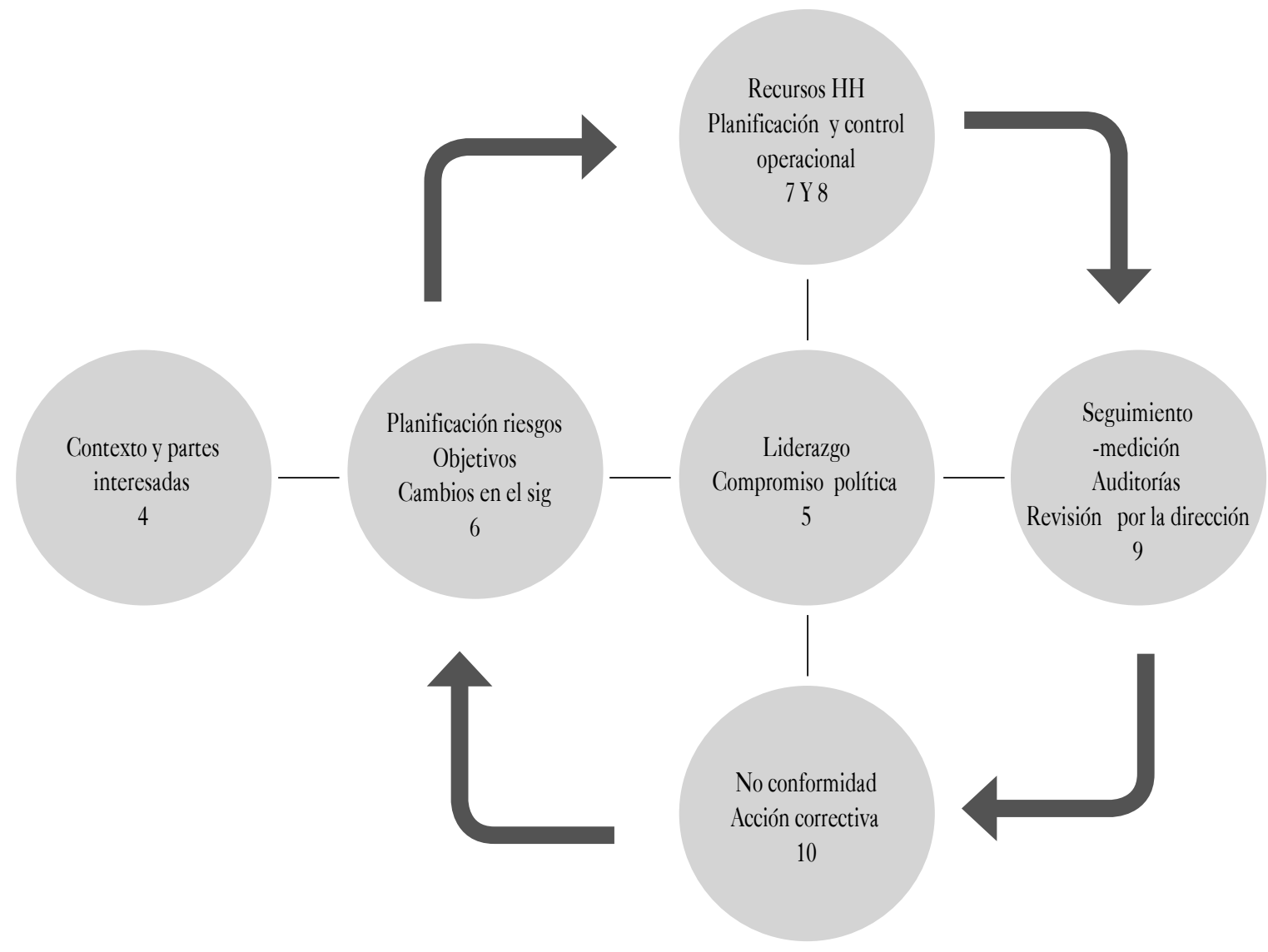

Fuente: elaboración propia.

La citada norma se fundamenta, entre otros, en los siguientes principios para la gestión de la calidad:

i) Enfoque al cliente. La gestión de la calidad debe orientarse principalmente a cumplir los requisitos del cliente y a tratar de exceder sus expectativas.

ii) Compromiso de las personas. Las personas competentes, empoderadas y comprometidas son esenciales para aumentar la capacidad y para generar valor.

iii) Enfoque a procesos. Se alcanzan resultados previsibles, eficaces y eficientes cuando las actividades se gestionan como procesos interrelacionados que funcionan como un sistema; iv) Toma de decisiones basada en la evidencia. Las decisiones basadas en datos e información tienen mayor probabilidad de producir los resultados deseados.

v) Mejora. Las organizaciones con éxito tienen un enfoque continuo hacia la mejora.

La teoría con mayor afinidad y correlación con el sistema de gestión de la calidad basada en el modelo NTC ISO 9001:2015 es la teoría de la creación del conocimiento de Nonaka y Takeuchi (Camison-Zornoza, BoronatNavarro, Villar-López, \& Puig-Denia, 2009). Esta teoría considera las organizaciones como sistemas sociales abiertos que interactúan con su entorno, donde se analiza la conversión del conocimiento tácito que poseen 
las personas y su compromiso para convertirlo en conocimiento explícito, orientado a mejorar resultados en cuanto a las relaciones con los clientes.

El modelo de la norma técnica NTC ISO 9001:2015 tiene tres partes claramente definidas en relación con el conocimiento que se gestiona en una organización. En una primera parte, existe un conocimiento que debe ser identificado: (i) la organización y su contexto, (ii) las necesidades y expectativas de las partes interesadas y (iii) los requisitos del cliente. En una segunda parte, este conocimiento debe ser procesado por la organización para generar más conocimiento. En la última parte, el conocimiento debe ser explotado para (i) obtener productos y servicios y (ii) satisfacer a las partes interesadas (figura 2).

Figura 2. Partes integrantes del conocimiento en las organizaciones

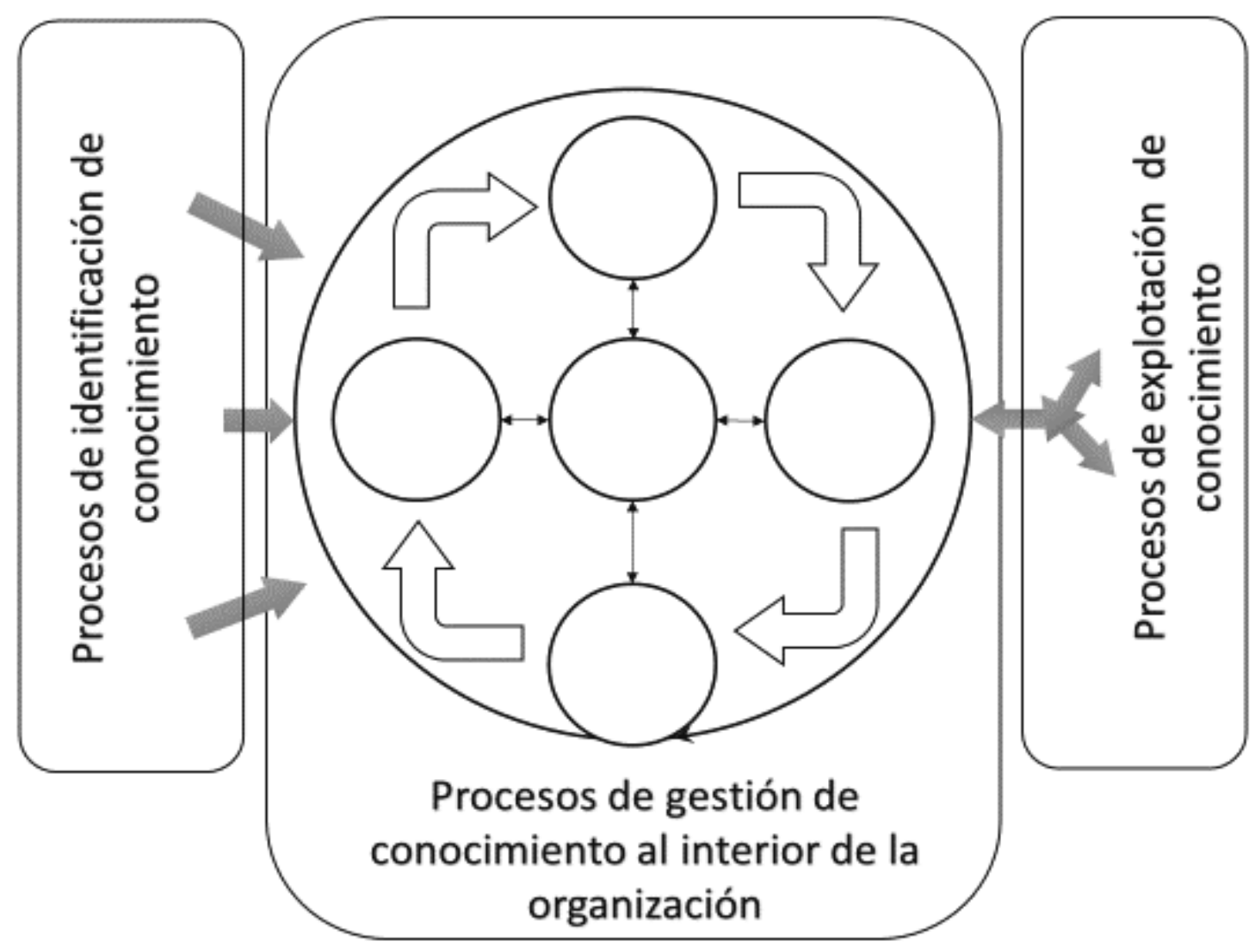

Fuente: elaboración propia.

Teniendo en cuenta lo anterior, debe establecerse cómo debe gestionar la empresa el conocimiento. En este sentido, de las múltiples teorías asociadas con gestión del conocimiento ya expuestas, la denominada "Empresa creadora de conocimiento" de Nonaka y Takeuchi es la que más brinda herramientas para diseñar un modelo al interior de las organizaciones; según estos autores, el conocimiento es "un proceso humano y dinámico de justificación de las creencias personales para perseguir la verdad". La creación del conocimiento se inicia a nivel individual como una "creencia verdadera justificada" y luego, a través de las interacciones sociales de 
los individuos, estas creencias se consolidan y convierten en un conocimiento compartido a nivel de la organización. El modelo se fundamenta teóricamente en dos dimensiones: una epistemológica y otra ontológica, estableciéndose entre ellas una interacción dinámica; la dimensión epistemológica se desagrega en conocimiento tácito y conocimiento explícito, mientras que en la dimensión ontológica el conocimiento se desarrolla a nivel individual, grupal, organizacional e interorganizacional. La interacción entre estas dos dimensiones se esquematiza en la denominada "espiral del conocimiento".

\section{La empresa creadora de conocimiento}

Ikujiro Nonaka (1994) ha desarrollado y propuesto un modelo de gestión del conocimiento, basado en la existencia de dos tipos de conocimiento: el conocimiento explícito y el conocimiento tácito. El conocimiento explícito puede ser expresado en palabras o números y puede ser rápidamente compartido y transmitido en forma de datos, fórmulas científicas, especificaciones, manuales y similares. Por otra parte, el conocimiento tácito es altamente personal, siendo muy complicado formalizarlo, comunicarlo o compartirlo. Hay dos dimensiones en el conocimiento tácito: la primera es de carácter técnico, lo que comúnmente se conoce como know-how; la segunda es la dimensión cognitiva, que consta de creencias, ideales, valores, esquemas y modelos mentales que están profundamente inmersos en cada persona.

El modelo es un proceso en espiral de interacción entre conocimiento explícito y conocimiento tácito, comúnmente denominado SECI, por las iniciales de los patrones que maneja: socialización, externalización, combinación e internalización. La combinación de las dos categorías hace posible la conceptualización de cuatro patrones de trasmisión del conocimiento.

La socialización es el primer patrón, que conlleva el intercambio de conocimiento tácito entre individuos; se basa en procesos de empatía y en compartir experiencias de vida. En la práctica, la socialización involucra procesos de captura de conocimiento a través de la proximidad física. Un segundo patrón es la externalización, donde el conocimiento tácito se vuelve explícito; es decir, el conocimiento se traduce a formas comprensibles que pueden ser entendidas por otros. Durante la etapa de externalización el individuo se integra a un grupo y se vuelve uno con el grupo. La combinación es el tercer patrón, que involucra la conversión de un conocimiento explícito en formas más complejas de conocimiento explícito, en esta etapa son claves los procesos de comunicación, difusión y sistematización de conocimiento. En la práctica, la fase de combinación conlleva tres procesos: (i) captura e integración de nuevo conocimiento explicito; (ii) diseminación de conocimiento explícito y (iii) edición y proceso del conocimiento explícito para que sea más útil para la organización (i.e., documentos como planes, reportes, datos del mercado, etc.). El cuarto patrón es la internalización, que hace referencia a convertir conocimiento explícito en conocimiento tácito. Aquí el individuo hace suyo el conocimiento de la organización. En la práctica la internalización posee dos dimensiones: la primera conlleva la actualización de conceptos relacionados con estrategia, táctica, innovación o mejoras en la organización; la segunda conlleva procesos de aprendizaje personal como aprender haciendo simulaciones o experimentos.

Para que estos procesos se puedan dar de mejor forma, Nonaka y Konno (1998) traen el concepto de Ba, originalmente propuesto por el filósofo japonés Kitaro Nishida y desarrollado posteriormente por H. Shimizu. El $B a$ puede asimilarse a un espacio compartido donde emergen relaciones, espacio que puede ser físico, virtual, mental o cualquier combinación de ellos. Nonaka y Konno (1998) consideran que el conocimiento se encuentra integrado en este tipo de espacios, existiendo en muchos niveles en una organización y estando conectados entre sí.

Los cuatro tipos de espacios $(B a)$ corresponden a las cuatro etapas del modelo SECi. Cada espacio soporta un 
proceso de creación del conocimiento. Hay un espacio que se denomina de origen, el primero, donde los individuos comparten sentimientos, emociones, experiencias y modelos mentales, que representa la fase de socialización; los elementos organizacionales que más estrechamente se relacionan en este espacio son la visión y la cultura. Un segundo espacio, el de interacción, es el lugar donde el conocimiento tácito se hace explícitofase de externalización-y donde el diálogo es el componente clave para la transformación de conocimiento. El espacio cibernético, el tercero, se asocia a la fase de combinación. Este espacio cibernético es el lugar donde se genera y sistematiza nuevo conocimiento explícito, junto con la información y el conocimiento explícito existente. El sistema documental es el soporte en este tipo de espacios. Por último, el espacio de ejercicio soporta la fase de internalización del conocimiento, donde el uso del conocimiento formal (explícito) en la vida real o en aplicaciones simuladas permite que el conocimiento se interiorice en las personas. La figura 3 muestra los procesos relacionados con la creación de conocimiento.

Figura 3. Fases de la creación del conocimiento. Modelo SECI

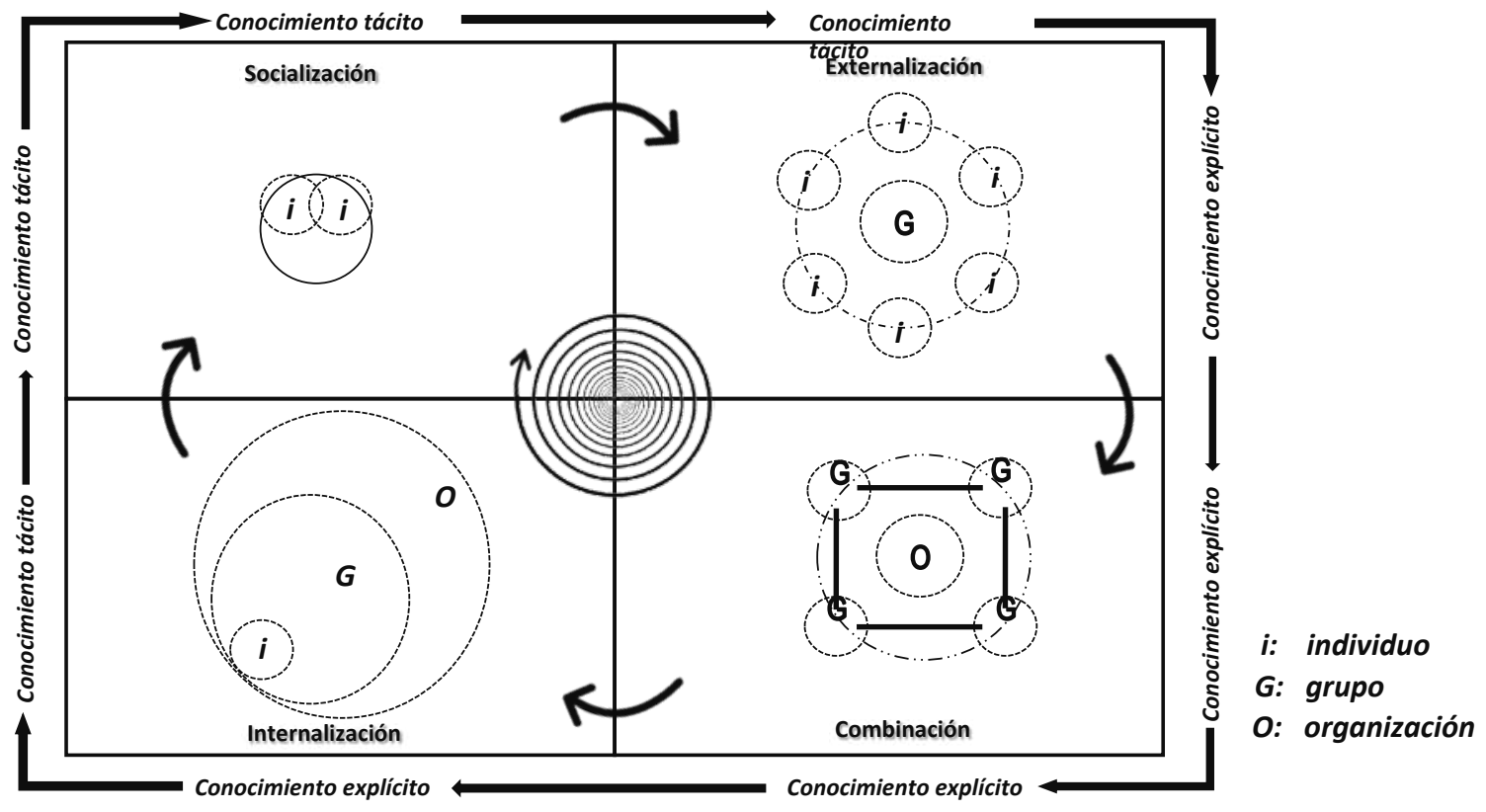

Fuente: elaboración propia con base en Nonaka y Konno (1998).

Al considerar estos espacios en los procesos de calidad, puede asimilarse que la etapa de planear involucra emplear los resultados de los procesos de identificación de conocimiento - de la organización y su contexto, de las partes interesadas y del cliente- y también del conocimiento resultado de los procesos del ciclo PHV. Aquí se comparten sentimientos, emociones, experiencias y modelos mentales, desde la organización, la visión y la cultura. Esta etapa estaría muy relacionada con la fase de socialización y un espacio de origen la soportaría.

Por otra parte, la etapa de hacer conlleva la implementación de lo planeado, es decir, se convierte el conocimiento tácito en explícito, como en la fase de 
externalización de modelo SEcI. Un espacio de interacción soportaría esta etapa.

La etapa de verificación conlleva realizar seguimiento, medición, análisis y discusión de los resultados, teniendo como referentes la información y el conocimiento previamente generado. Nótese que esta etapa se relaciona con la fase de combinación, correspondiente a la interacción de nuevo conocimiento explícito con información y conocimiento existente, generando y sistematizando conocimiento explícito en toda la organización. El espacio cibernético corresponde al espacio de interacción de esta fase del conocimiento.

Por último, el actuar conlleva tomar acciones para mejorar, lo que implica aprender e interiorizar el conocimiento explícito; es decir, es la fase de internalización del conocimiento que puede soportarse por el espacio de ejercicio.

\section{Modelos de Gestión del Conocimiento para pymes del Comité Europeo de Normalización y modelo español derivado}

El Comité Europeo de Normalización (CEN) publicó en marzo del 2004 una serie de Normas (CWA 14924) como guía para las buenas prácticas en gestión del conocimiento. Estas cinco normas están enfocadas en (1) establecer un marco sobre la gestión del conocimiento; (2) explicar cómo crear una cultura para introducir gestión del conocimiento; (3) suministrar elementos para implementar gestión del conocimiento en pequeñas y medianas empresas; (4) medir la gestión del conocimiento y (5) explicar la terminología asociada a la gestión del conocimiento. Las normas buscan llevar a las pequeñas y medianas empresas la gestión del conocimiento como elemento de competitividad. El modelo CEN tiene tres capas, según se observa en la figura 4 :

Fiǵura 4. Modelo CEN para la gestión del conocimiento

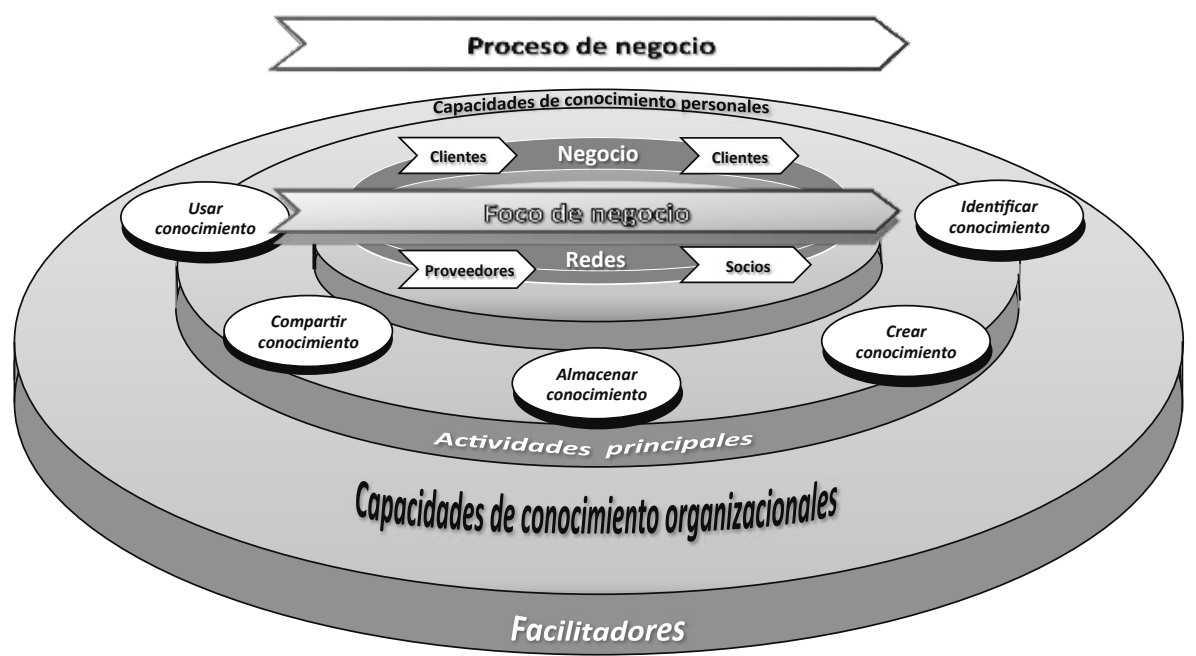

Fuente: elaboración propia basada en la norma CWA 14924. 
La primera capa se centra en el eje del negocio, que debe estar en el centro de cualquier iniciativa de gestión del conocimiento. Representa los procesos de valor agregado de la organización que incluyen, típicamente, el desarrollo de una estrategia, desarrollo de un productol servicio, fabricación, entrega, ventas y soporte al cliente (figura 5). Estos procesos representan el contexto organizacional en el que el conocimiento crítico —como el conocimiento acerca de productos, servicios, clientes 0 tecnología - es creado y aplicado. Esto procesos involucran a aquellos que interactúan con la organización, como proveedores, socios y clientes.

Fiǵura 5. Modelo CEN para la gestión del conocimiento. Enfoque estratégico

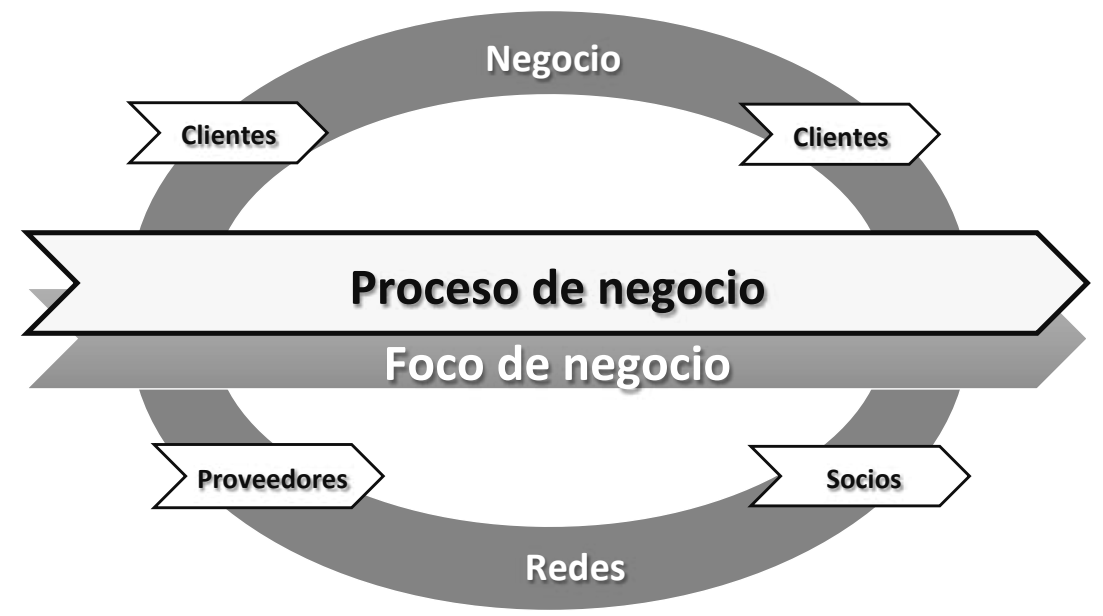

Fuente: elaboración propia con base en la norma CWA 14924.

La segunda capa está relacionada con las actividades de gestión del conocimiento que han sido identificadas como las más ampliamente usadas en Europa (figura 6): (i) identificar conocimiento, (ii) crear conocimiento, (iii) almacenar conocimiento, (iv) compartir conocimiento y (v) usar conocimiento. Estas actividades tradicionalmente soportan el eje del negocio y los procesos de negocio.

Figura 6. Modelo cEN para la gestión del conocimiento. Actividades de gestión

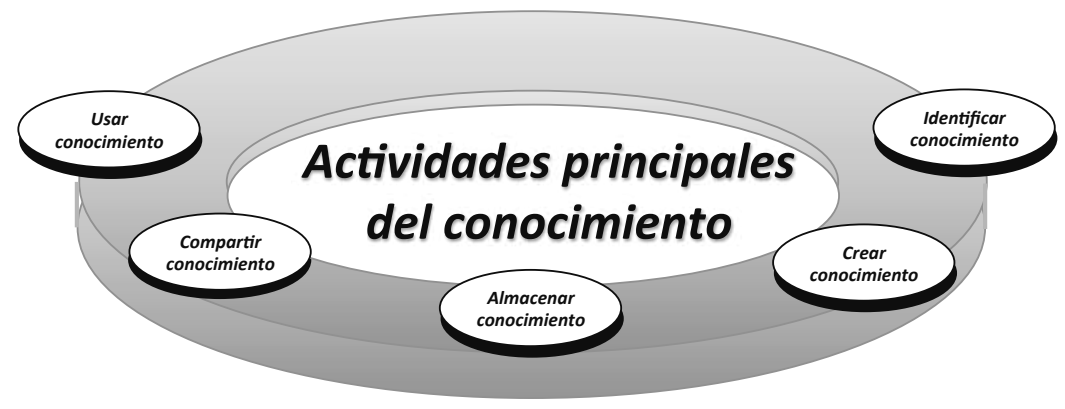

Fuente: elaboración propia con base en la norma CWA 14924. 
Por último, se encuentran los facilitadores que conforman la tercera capa y comprenden dos categorías principales que se complementan (figura 7). La primera, el conocimiento personal, incluye todas las capacidades de las personas, tales como ambiciones, destrezas, creencias o experiencias que pueden ser desarrolladas a nivel personal o grupal para generar mejoras desde el manejo del conocimiento. La segunda, las capacidades de conocimiento organizacional, son aquellas que los líderes tienen que establecer para permitir un manejo efectivo del conocimiento dentro de procesos de valor agregado, desde partes interesadas internas (i. e., gerentes y empleados) y socios externos (i. e., clientes y proveedores). Estas capacidades incluyen misión, visión, estrategia, diseño de procesos y estructuras organizacionales, como también el desarrollo de conocimiento colectivo de una organización —activos del conocimiento-.

Figura 7. Modelo cEN para la gestión del conocimiento. Facilitadores

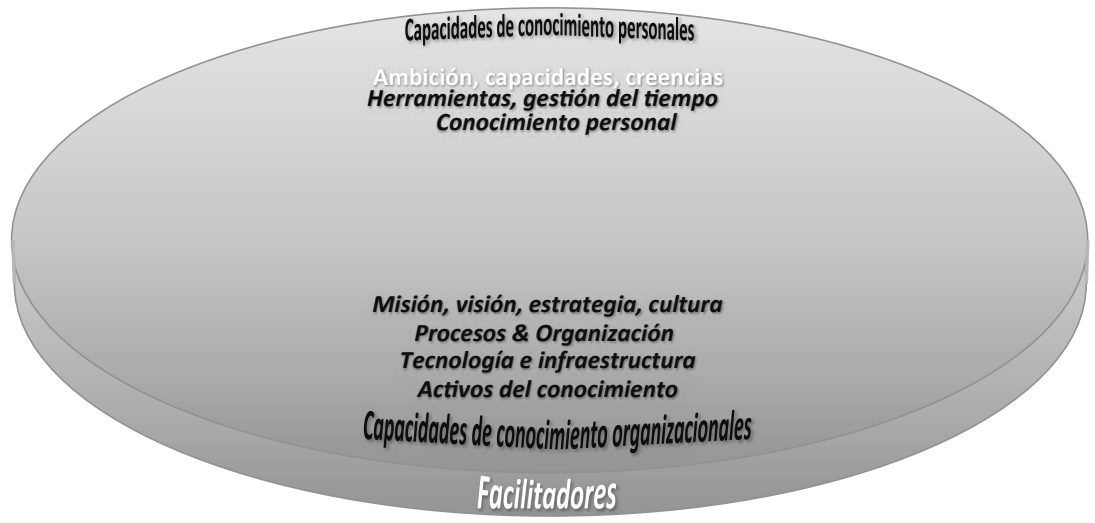

Fuente: elaboración propia con base en la norma CWA 14924.

La norma uNE 412001 de diciembre de 2008, Guía práctica de gestión del conocimiento (Asociación Española de Normalización [AENOR], 2008), parte del modelo CEN para la creación de un modelo propio (modelo español). El modelo español constituye el marco conceptual que recoge planteamientos metodológicos, referencias a casos y herramientas que facilitan y orientan el desarrollo de proyectos de gestión del conocimiento, y su estructura general se observa en la figura 8 .

Fiǵura 8. Modelo une 412001 para la gestión del conocimiento

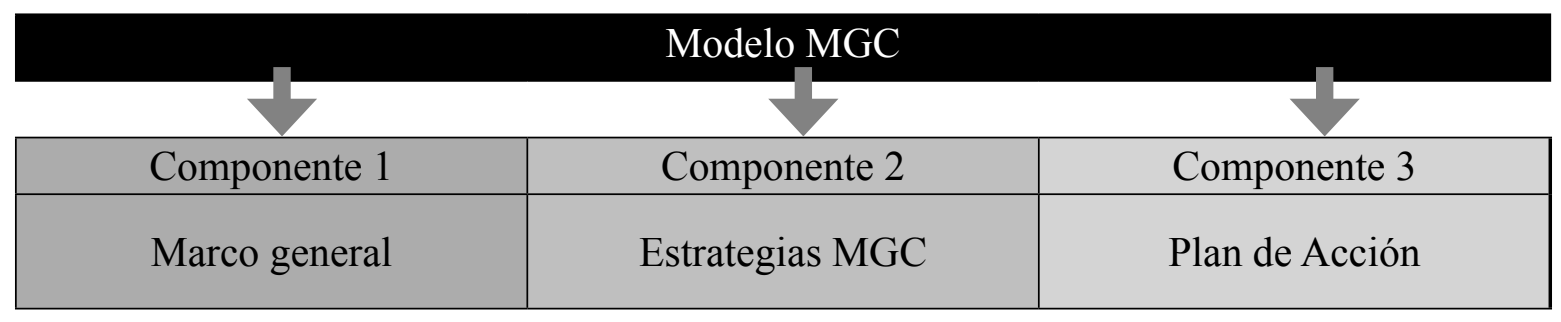

Fuente: adaptada de AENOR (2008). 
Debe notarse la similitud con las tres capas propuestas por el modelo CEN. En el marco de este modelo, deben destacarse las prácticas de gestión de conocimiento que se han de implantar agrupadas en un enfoque, tal y como se ejemplifica en la figura 9, en un flujo de conocimiento.

Figura 9. Prácticas de gestión del conocimiento según UNE 412001

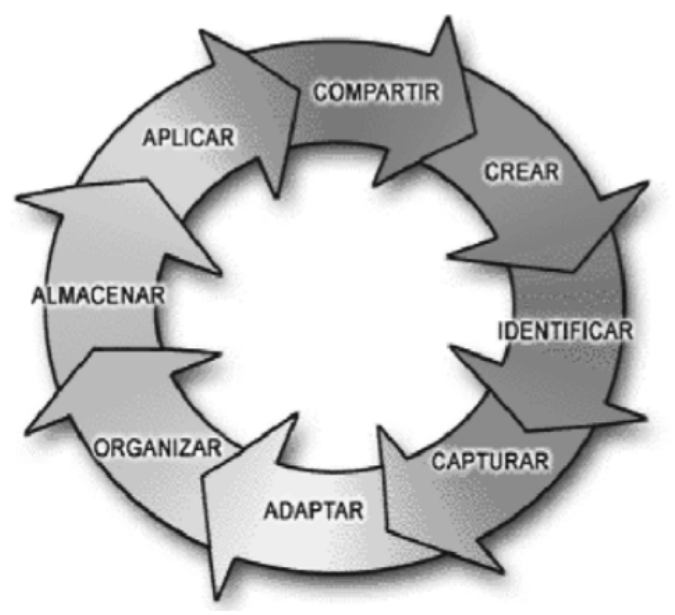

Fuente: adaptada de AENOR (2008).

En este punto es importante notar que, en los modelos CEN y español, la primera y tercera capas -0 primer y tercer componente- están muy ligadas a procesos dentro de la organización, y la segunda capa —o segundo componente- es propia de la gestión del conocimiento. Consecuentemente, puede considerarse tomar esta segunda capa —o segundo componente- e introducirlo en un sistema de gestión de calidad por procesos, como la NTC ISO 9001:2015, que maneja de una manera más acertada la primera y tercera capas - 0 primer y tercer componente- - De esta forma, se puede diseñar un modelo más simple y más aplicable a pymes.

\section{La gestión documental como soporte de la gestión del conocimiento}

Tal y como señalan Álvarez-Álvarez et al. (2013), la documentación resulta muy importante en una organización, así como su gestión, debido a cuatro razones fundamentales:
- Permite y facilita la comunicación del sistema de gestión con todas las partes interesadas (proveedores, empleados, clientes, gobierno, accionistas, etc.).

- Suministra un marco de operación claro y eficiente, pues permite declarar la forma en que se llevarán a cabo las actividades.

- Provee evidencia objetiva de cómo se alcanzan los resultados, y genera una base para evaluar y mejorar la eficacia y adecuación continua del sistema de gestión de la calidad.

- Es la base para la formación inicial y la actualización periódica de los empleados.

La gestión de los documentos se relaciona con el diseño normalizado de los documentos, el control tanto de la duplicidad como de versiones, la simplificación, el control y uso de procedimientos, la adecuada organización e implementación de los procesos archivísticos de clasificación, ordenación y descripción de la documentación de una organización, así como la conservación, valoración, disponibilidad para la toma de decisiones y eliminación de documentos que carecen de valor para la gestión. Lo anterior conlleva, en la práctica, la fijación de políticas y normas, la asignación de responsabilidades, el establecimiento de procesos y procedimientos para que un documento sirva de soporte auténtico, con fiabilidad, integridad y de conformidad con las actividades propias de la entidad, que garantice la continuidad de las actividades, además del cumplimiento de las normas legales.

La norma técnica ISo 30301:2011 hace referencia a esta integralidad en lo relacionado con procesos y controles para los documentos desde su creación, donde el requisito fundamental es que los documentos permanezcan accesibles en el tiempo que sea necesario. La norma en este sentido es muy clara cuando requiere de la implementación de una política y unos objetivos de gestión 
documental y la definición de controles desde el origen del documento hasta su destino final, con el fin de facilitar su consulta, conservación y utilización para la toma de decisiones.

La gestión de los documentos es parte integral de las actividades de las organizaciones, en dirección hacia la eficiencia, la eficacia y la preservación de la memoria institucional. Tal y como se señalan Álvarez-Álvarez et al. (2013), existe una falta de interacción entre la gestión de la calidad y la gestión documental, y frecuentemente son vistos como sistemas independientes, lo que no solo incide negativamente sobre el desempeño organizacional, sino que impide que se capitalice el conocimiento que se genera y limita la formulación de propuestas innovadoras.

\section{Enfoque basado en procesos para los sistemas de gestión}

De acuerdo con la ISO (2003):

Un enfoque basado en procesos es una excelente vía para organizar y gestionar la forma en que las actividades de trabajo crean valor para el cliente y otras partes interesadas. Las organizaciones están estructuradas a menudo como una jerarquía de unidades funcionales. Las organizaciones habitualmente se gestionan verticalmente, con la responsabilidad por los resultados obtenidos dividida entre unidades funcionales. El cliente final u otra parte interesada no siempre ve todo lo que está involucrado. En consecuencia, a menudo se da menos prioridad a los problemas que ocurren en los límites de las interfases que a las metas a corto plazo de las unidades. Esto conlleva a la escasa o nula mejora para las partes interesadas, ya que las acciones están frecuentemente enfocadas en las funciones más que en el beneficio global de la organización. El enfoque basado en procesos introduce la gestión horizontal, cruzando las barreras entre diferentes unidades funcionales y unificando sus enfoques hacia las metas principales de la organización. También mejora la gestión de las interfases del proceso. (p. 4)
En este mismo sentido, Mallar (2010) señala lo siguiente:

El modelo de Gestión basada en los Procesos, se orienta a desarrollar la misión de la organización, mediante la satisfacción de las expectativas de sus stakeholders -clientes, proveedores, accionistas, empleados, sociedad,.- y a qué hace la empresa para satisfacerlos, en lugar de centrarse en aspectos estructurales como cuál es su cadena de mandos y la función de cada departamento. Así, define proceso como un conjunto de actividades de trabajo interrelacionadas, que se caracterizan por requerir ciertos insumos (inputs: productos o servicios obtenidos de otros proveedores) y actividades específicas que implican agregar valor, para obtener ciertos resultados (outputs). (p. 44)

Además, Mallar (2010) establece los elementos que conforman un proceso:

1. Inputs: pueden ser recursos por transformar, materiales que procesar, personas por formar, informaciones que procesar, conocimientos que elaborar y sistematizar, etc.

2. Recursos o factores que transforman, los cuales actúan sobre los inputs: aquíse encuentran (i) elementos humanos -que planifican, organizan, dirigen y controlan las operaciones- y (ii) elementos de apoyo - como la infraestructura tecnológica一.

3. Flujo de procesamiento o transformación: la transformación puede ser física, de lugar, pero también puede modificarse una estructura jurídica de propiedad. Si el input es información, se puede tratar de reconfigurarla (como en servicios financieros), 0 posibilitar su difusión (comunicaciones). Puede también tratarse de la transferencia de conocimientos. A su vez, se puede actuar sobre el mismo cliente de forma física, transportarlo, dársele alojamiento o actuar sobre su cuerpo o en su psicología y satisfacción.

4. Outputs: son básicamente de dos tipos: (i) bienes: tangibles (ii) servicios: intangibles. 
Teniendo en cuenta lo indicado por la Iso y por Mallar (2010), es conveniente que un modelo de gestión del conocimiento dirigido a pymes se ajuste a un enfoque basado en procesos.

La norma IS0 9001:2015, basada en un enfoque en procesos, requiere unas fuentes de entrada, unas entradas, unas actividades, unas salidas y unos receptores de las salidas, como se muestra en la figura 10. Las entradas y las salidas se basan en materia, energía o información. El conocimiento, información con sentido, en consecuencia, es la base de los procesos de los sistemas de gestión. Más relevante aún es el hecho de que procesos de gestión del conocimiento sirven para fortalecer los procesos de sistemas de gestión de calidad.

\section{Prácticas de gestión documental en pymes}

A través de la realización de cuatro proyectos de grado desarrollados por estudiantes del programa Sistemas de Información y Documentación, de la Universidad de La Salle, en los cuales se aplicó una entrevista a 47 pymes de cuatro sectores en tres municipios, se concluyó que en relación con la gestión documental las pymes no tienen conceptos claros con respecto a la gestión documental, solo conservan los documentos propios de la gestión comercial (Castillo, 2015) y consideran la gestión documental un gasto, no una inversión (Martínez, 2015); por otra parte, Rodríguez (2015) plantea que existe una ausencia total de técnicas, herramientas y procesos relacionados con la gestión documental. La adopción de un modelo de gestión documental debe estar asociado a la asignación de recursos físicos y humanos, y a una planeación desde la gerencia para obtener sus beneficios (Barrera, 2015). Las pymes estudiadas no consideran la gestión de la calidad relevante para su actividad económica, pero no descartan su implementación hacia el futuro.
Con respecto a la gestión del conocimiento, las pymes no tienen claridad sobre su importancia como valor estratégico para el desarrollo de productos, procesos y nuevos mercados que contribuyan a la competitividad de la empresa (Castillo, 2015). Las anteriores conclusiones establecen un panorama desalentador en cuanto a la gestión que llevan a cabo las pymes, que operan solo para sobrevivir, pero al mismo tiempo crea una gran oportunidad para plantear soluciones viables para robustecer su desempeño.

\section{Propuesta de un modelo para la gestión del conocimiento en pymes}

Un sistema de gestión del conocimiento basado en un enfoque por procesos se cimienta en el conocimiento individual de las personas de la organización y en el conocimiento capturado del exterior. Este conocimiento se utiliza para llevar a cabo las operaciones y funciones propias de la organización, que se deben planificar como procesos, constituyéndose estos como el eje articulador de la gestión de la calidad, la gestión documental y la gestión del conocimiento.

Al usar el conocimiento, este se comparte de forma tácita entre las personas y, para que se mejore, debe ser documentado, con el fin de conservarlo, que sirva de soporte y sea usado en los procesos de la organización. Luego de la transformación, el conocimiento debe ser generador de valor y debe ser apropiado a nivel individual, para que sirva de insumo en un nuevo proceso de gestión del conocimiento.

Así, un proceso de gestión del conocimiento puede soportar un proceso de un sistema de gestión de la calidad. En la figura 11 se expone el modelo propuesto basado en el sistema de gestión de la calidad y gestión documental. 


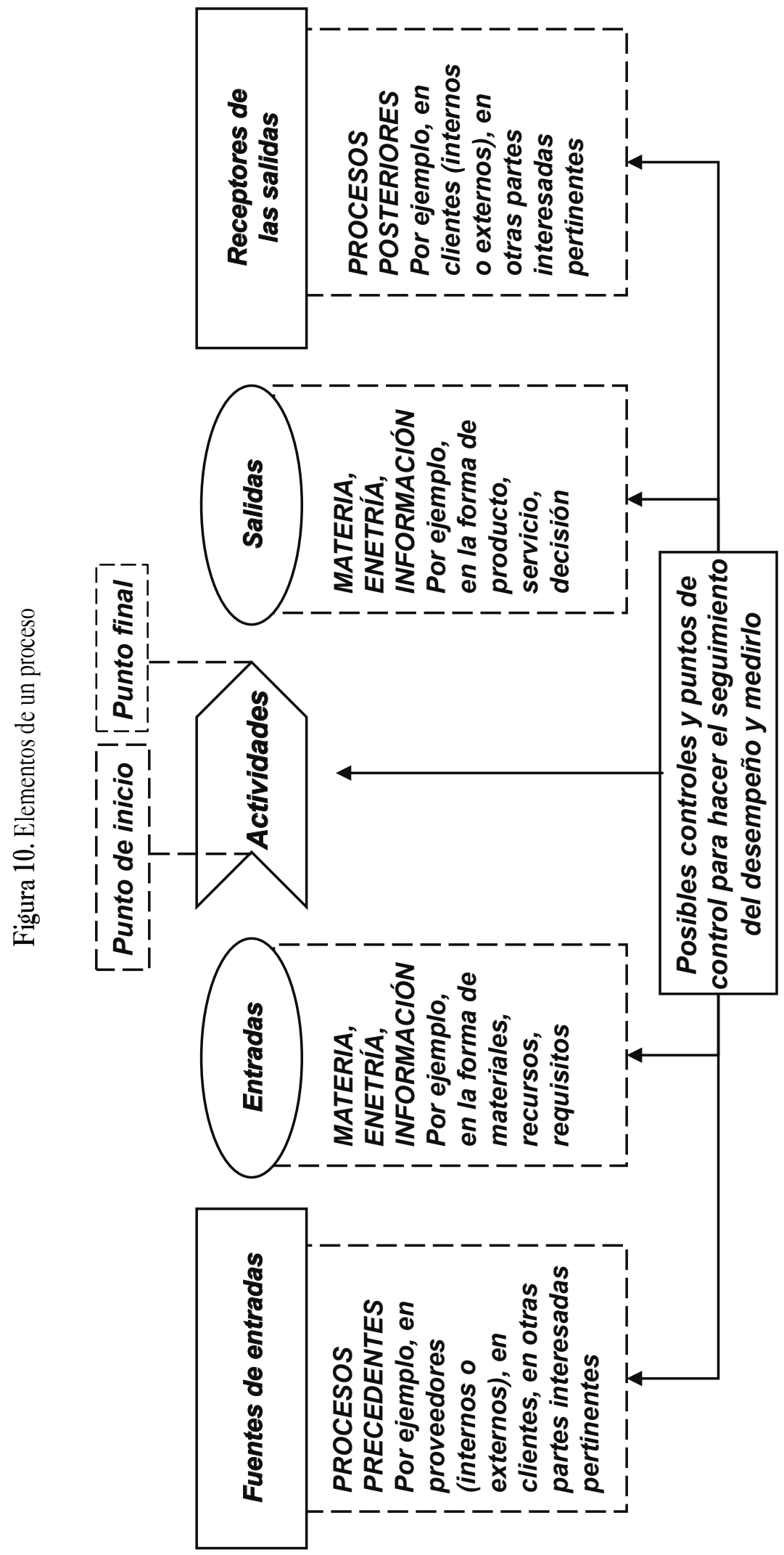



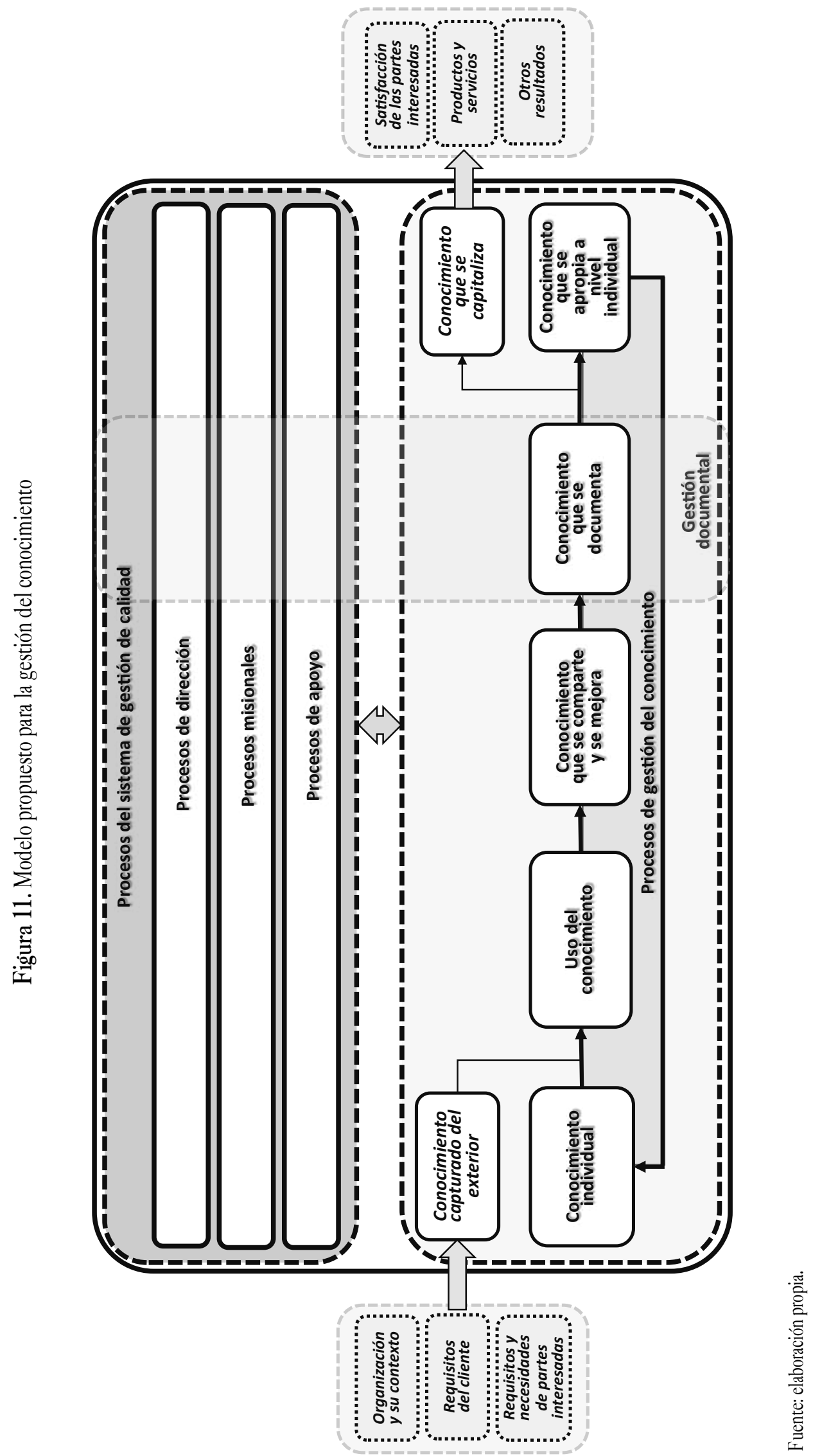
En el caso de un proceso de gestión basado en la NTC ISO 9001:2015, el conocimiento capturado del exterior se relaciona con (i) la organización y su contexto, (ii) los requisitos de cliente y (iii) las necesidades y expectativas de las partes interesadas.

Este conocimiento capturado del exterior, junto con el conocimiento individual de las personas vinculadas a la organización, debe ser empleado para su aprovechamiento al interior de la organización. En este sentido, lo indicado en los modelos CEN y español permiten materializarlo dentro de la organización. Este conocimiento capturado debe ser compartido a los demás miembros de la organización y mejorado a partir de las experiencias individuales. Así es como el conocimiento tácito se vuelve explícito. Este proceso al interior de la organización se fundamenta en el ciclo PHA — planear, hacer, verificar, actuar-, que soporta los procesos de dirección, misionales y de apoyo. Pero este conocimiento generado debe capitalizarse, por medio de la satisfacción del cliente, productos y servicios generados y resultados que incluyen el cumplimiento de lo esperado por las partes interesadas en el proceso.

Pero la capitalización también se encuentra en la apropiación individual del conocimiento trabajado. Entonces, ese conocimiento explícito se vuelve tácito para iniciar un nuevo proceso. Nótese que este proceso corresponde al modelo SECI de Nonaka, en el que juegan un papel muy importante diferentes tipos de espacios creados al interior de la organización. Así, se requieren espacios de origen, donde los miembros compartan sentimientos, emociones, experiencias, modelos mentales y, no menos importante, visión y cultura. Un espacio de interacción donde el diálogo es el componente clave para la transformación de conocimiento. El espacio cibernético, el tercero, es el lugar donde se genera y sistematiza nuevo conocimiento explícito, junto con información y conocimiento explícito existente. El sistema documental, la familia de normas ISO 30300 y los procesos de gestión documental serán el soporte de estos espacios, permitiendo que el conocimiento quede de forma explícita en la organización. Por último, un espacio de ejercicio permite que el conocimiento transformado se interiorice en las personas.

Todo este proceso de gestión del conocimiento soporta, a su vez, procesos de dirección, misionales y de apoyo. Los procesos directivos son aquellos a través de los cuales una empresa o una dirección conjunta de una red planifican, organizan, dirigen y controlan recursos, proporcionando el direccionamiento a los demás procesos. Los procesos misionales, por su parte, se orientan al logro del propósito organizacional y a su visión. Los procesos de apoyo son aquellos servicios internos necesarios para realizar los procesos del negocio (Mallar, 2010).

Por último, el conocimiento transformado a lo largo de la organización debe ser capitalizado en varias vías; debe satisfacer a las partes interesadas; puede permitir la generación de nuevos productos y servicios, y puede permitir la obtención de activos intangibles, entre muchos otros logros. El modelo permite una adaptación rápida a organizaciones que posean un modelo de gestión NTC ISO 9001:2015 implementado, aun cuando no es un necesario para su implementación.

Asimismo, a pesar de la simplicidad del modelo, este es robusto y permite su implementación rápida en organizaciones de todos los tamaños, especialmente en pequeñas y medianas empresas, puesto que la identificación de los procesos por medio de los cuales la organización cumple su misión, así como los procesos de apoyo y los de direccionamiento, se constituye en un primer acercamiento a la formalidad de la estructura organizacional, que es una de las deficiencias normales en las pymes. Además, la documentación a través de los procesos, que captura el conocimiento, lo preserva, consolida las mejoras y facilita su transmisión, crea las bases para cimentar una cultura administrativa menos empírica y mejor fundamentada. 


\section{CONCLUSIONES}

Tras haber hecho un análisis de la norma NTC ISO 9001:2015, en relación con la gestión del conocimiento, y al haber analizado diferentes modelos de gestión del conocimiento - dentro de los que destaca el modelo CEN, el modelo español y el modelo de Nonaka一, se ha propuesto un modelo de gestión del conocimiento simple y, a su vez, robusto que incorpora la gestión documental. Este modelo procura ser de fácil aplicación en las organizaciones, especialmente las pequeñas y medianas empresas, apoyando la administración general y brindando la red de procesos como un elemento de base para formalizar la estructura organizacional.

El modelo propuesto involucra un proceso de captura de conocimiento y un esquema simplificado de los modelos CEN y uNE, que está en consonancia con lo establecido por el modelo SECI de Nonaka y Takeuchi. Uno de estos espacios se encuentra soportado en las normas de gestión documental Iso 30301 y es transversal a todos los procesos. Todo el modelo soporta los procesos de dirección, misionales y de apoyo de la institución. Asimismo, los resultados de los procesos asociados al modelo son los mismos esperados en la norma técnica NTC ISO 9001:2015: satisfacción de las partes interesadas, nuevos productos o servicios y generación de otros activos intangibles. Finalmente, se requieren estudios empíricos que direccionen la confiabilidad del modelo y establezcan su adaptabilidad en las pymes.

\section{REFERENCIAS}

Asociación Española de Normalización (AENOR). (2008). Norma UNE 412001. Guía Práctica de Gestión del Conocimiento. Madrid, España.

Álvarez-Álvarez, M. J., Castro-Rojas, M., \& Peña G. (2013). Gestión del conocimiento: una perspectiva desde la gestión de la calidad y la gestión documental. Signos - Investigación en Sistemas de Gestion, 5(2), 101-118.

Asociación Nacional de Instituciones Financieras (ANIF). (2017a). Gran encuesta Pyme. Recuperado de http:// www.anif.co/sites/default/files/publicaciones/gepnacional_ii-17.pdf

Asociación Nacional de Instituciones Financieras (ANIF). (2017b). Comentario Económico del día - Anif. Recuperado de de http://anif.co/sites/default/files/ago1417.pdf

Banco Mundial. (2015). Topics. Recuperado de de http:// www.worldbank.org/en/topic/financialsector/brief/ smes-finance

Barrera, J. A. (2015). Análisis de la gestión documental en el sector bebidas y alimentos. Pymes de cinco localidades de Bogotá, (tesis de pregrado). Universidad de La Salle, Bogotá.

Camison-Zornoza, C., Boronat-Navarro, M., VillarLópez, A., \& Puig-Denia, A. (2009). Sistemas de gestión de la calidad y desempeño: importancia de las prácticas de gestión del conocimiento y de I+D. Revista Europea de Dirección y Economía de la Empresa, 18(1), 123-134.

Castillo, A. (2015). La gestión documental en el sector de Pymes en el municipio de Mosquera, (tesis de pregrado). Universidad de La Salle, Bogotá.

Drucker, P. (1992). The New Society of Organizations. Harvard Business Review, Septiembre-Octubre. Recuperado de https://hbr.org/1992/09/the-new-societyof-organizations

Drucker, P. (1994). Post-capitalist society. New York: HarperCollins Publishers.

Estas son las pymes más ganadoras de Colombia en 2017. (14 de septiembre de 2017). Revista Dinero. 
Recuperado de de http://www.dinero.com/edicionimpresa/caratula/articulo/ranking-de-las-mejoresPymes-de-colombia-en-2017/249828

Ferrás, X. (2016). Blog de Xavier Ferrás. Recuperado de de http://xavierferras.blogspot.com.co/2016/05/lacuarta-revolucion-industrial.html

Flaherty, J. E. (2001). Peter Drucker: La esencia de la administración moderna. Naucalpan de Juaréz, México: Pearson Educación.

Instituto Colombiano de Normas Técnicas y Certificación (Icontec). (2015). Norma Técnica Colombiana NTC-ISO 9001. Sistemas de gestión de la calidad. Bogotá: Icontec.

International Organization for Standardization (ISO). (2011). Iso 30301. Information and documentation Management systems for records - Requirements. Ginebra: ISO.

International Organization for Standarization (ISO). (2003). Orientación sobre el concepto y uso del "Enfoque basado en procesos" para los sistemas de gestión. Ginebra: ISO.

International Organization for Standardization (ISO). (2015). ISO 9001. Quality Management Systems - Requirements. Ginebra:ISO.

Mallar, M. Á. (2010). La gestión por procesos: un enfoque de gestión eficiente. Visión del futuro, 13(1). Recuperado de https://www.redalyc.org/ pdf/3579/357935475004.pdf
Martínez, A. (2015). La gestión documental en las Pymes del sector salud. laboratorios clínicos ubicados en la localidad 18 Rafael Uribe Uribe, (tesis de pregrado). Universidad de La Salle, Bogotá.

Nonaka, I. (1994). A dynamic theory of organizational knowledge creation. Organization science, 5(1), 14-37. Recuperado de https://www.jstor.org/stable/2635068

Nonaka, I., \& Konno, N. (1998). The concept of "Ba": Building a foundation for knowledge creation. California Management Revierw, 40(3), 40-54. doi: https:// doi.org/10.2307/41165942

Organización para la Cooperación y el Desarrollo Económicos (OECD). (2014). Small businesses, job creation and growth: facts, obstacles and best practices. Recuperado de https://www.oecd.org/cfe/smes/2090740.pdf

Peña, G. (2017). Integración de la gestión del conocimiento con el sistema de Gestión de la Calidad NTC ISO 9001:2015. En A. Espejo-Martínez \& A. L. Ramos (Eds.), Investigación y Desarrollo Económico Administrativo (pp. 365-384). Campeche, México: Editorial TECCIS.

Rodríguez, D. (2015). Modelo de gestión documental para las pequeñas Pyme del municipio de Soacha, con énfasis en certificación NTC 6001, (tesis de pregrado). Universidad de La Salle, Bogotá. 